\title{
Deployment Characteristics of "The Edge" in Mobile Edge Computing
}

\author{
Meenakshi Syamkumar \\ University of Wisconsin-Madison \\ ms@cs.wisc.edu
}

\author{
Paul Barford \\ University of Wisconsin-Madison \\ pb@cs.wisc.edu
}

\author{
Ramakrishnan Durairajan \\ University of Oregon \\ ram@cs.uoregon.edu
}

\begin{abstract}
The envisioned capabilities of mobile edge computing are predicated on a delivery infrastructure with capacity, ubiquity, robustness, and capabilities to serve a country-wide user base. In this paper, we present an empirical study of key aspects of mobile edge infrastructure toward the goal of understanding their current characteristics and identifying future deployments. We start by analyzing a dataset of over $4 \mathrm{M}$ cell tower locations in the US. We evaluate the geographic characteristics of deployments and highlight how locations correspond to population density in major metropolitan areas and in rural areas. We also show how deployments have been arranged along highways throughout the US. Our analysis highlight areas where new deployments would be warranted. Finally, we analyze how cell tower deployments correspond to current major data center locations and assess how micro servers might be deployed to improve response times and to better serve customers.
\end{abstract}

\section{CCS CONCEPTS}

- Networks $\rightarrow$ Network design principles; Wireless access points, base stations and infrastructure; Mobile networks;

\section{KEYWORDS}

MEC, Cellular infrastructure, Data centers, Population analysis

\section{ACM Reference Format:}

Meenakshi Syamkumar, Paul Barford, and Ramakrishnan Durairajan. 2018. Deployment Characteristics of "The Edge" in Mobile Edge Computing. In MECOMM'18: ACM SIGCOMM 2018 Workshop on Mobile Edge Communications , August 20, 2018, Budapest, Hungary. ACM, New York, NY, USA, 7 pages. https://doi.org/10.1145/3229556.3229557

\section{INTRODUCTION}

Future mobile edge communications (MEC) systems will provide a foundation for new applications and services that dramatically outperform today's offerings. One of the central tenets of the envisioned MEC infrastructure follows a well-worn path-push the computational capability closer to users. This offers the opportunity to significantly reduce edge-to-core latencies for mobile devices that are currently on the order of hundreds of milliseconds [23].

Permission to make digital or hard copies of all or part of this work for personal or classroom use is granted without fee provided that copies are not made or distributed for profit or commercial advantage and that copies bear this notice and the full citation on the first page. Copyrights for components of this work owned by others than ACM must be honored. Abstracting with credit is permitted. To copy otherwise, or republish, to post on servers or to redistribute to lists, requires prior specific permission and/or a fee. Request permissions from permissions@acm.org.

MECOMM'18, August 20, 2018, Budapest, Hungary

(C) 2018 Association for Computing Machinery.

ACM ISBN 978-1-4503-5906-1/18/08 .. \$15.00

https://doi.org/10.1145/3229556.3229557
A starting point for our work is defining and understanding the edge of MEC infrastructure. First, we define the edge as the locations of cell towers that will act as the primary service infrastructure for mobile devices. ${ }^{1}$ Next, we extend this notion to include distances between cell towers and data centers that provide computational and storage services. We posit that characterization of current cell tower and data center deployments will establish a baseline for understanding MEC performance and for identifying how new infrastructure deployment can enhance performance.

In this paper, we report results of an analysis of mobile edge infrastructure in the US. Our focus is a crowd-sourced database of over $4 \mathrm{M}$ cell tower locations in the US from OpenCelliD [11]. We argue that while this data is not definitive, it is large enough to provide useful insights on cell tower deployments. Along with cell tower locations, we also consider population distribution datasets from Census Bureau [1] and data center locations from Internet Atlas [19] in our analyses. Our location-based analysis utilizes a Geographic Information System (GIS) to combine these data sets and address questions such as: what is the distribution of cell towers in different areas of the US?, what is the average number of residents or drivers per cell tower? what is the average distance from cell tower to data center? and what is the potential impact of deployment of micro servers colocated with cell towers?

Visual inspection of cell tower locations shows that deployments are prevalent in densely populated areas and along major thoroughfares. This aligns with the notion of assuring service to the largest number of users. We assess the populations served by cell towers in selected large Metropolitan Statistical Areas (MSAs), rural areas and roadways by dividing regions into Voronoi cells. We find that cell tower deployments in large urban areas are dense and relatively consistent in the number of residents that they serve. In contrast, deployments in rural areas are much more sparse and less consistent. Similarly, cell tower deployments along highways tend to be relatively dense near urban hubs but deployments becomes less dense along rural stretches although the gradient is different than in residential areas.

Our analysis of cell tower deployments relative to nearest data centers is intended to highlight how the edge in MEC infrastructure can be moved closer to users in order to improve performance. Our analysis shows that cell tower-to-data center distances in large MSAs today are typically less than 10 miles, while distances in rural areas are greater by a factor of 2 . This baseline serves as a reference for a tiered analysis of deployments of micro data centers that would be colocated with cell towers. We show how cell towerto-data center distance can be dramatically reduced by focusing on

\footnotetext{
${ }^{1}$ While WiFi hotspots are ubiquitous and valuable in broad MEC deployment, we focus on cellular infrastructure in this paper due to its broader range and ability to support mobility.
} 
the Voronoi cells associated with the largest number of residents first and then deploying successively in cells with lower population density.

\section{DATASETS}

\subsection{Cell Tower Dataset}

In this study, we use cell tower data from OpenCelliD [11]. This crowd-sourced dataset has information about $\sim 40 \mathrm{M}$ cell towers worldwide. The repository includes geographic locations, Mobile Network Operators (MNOs), Mobile Country Codes (MCCs) and cell tower type (e.g., LTE, CDMA). Since the focus of our study is to elucidate the deployment characteristics of the edge in the US, we employ the following two filters.

US boundary filtering. To eliminate cell towers whose geolocation is outside the US, we apply a two-step filtering based on US boundaries. First, given a $c s v$ file of cell tower geolocations, we create a shape file of the cell tower dataset using spatial analysis tool from ESRI's ArcGIS [7]. We then perform the boundary-based filtering with overlap tool in ArcGIS, which eliminates cell towers outside the US boundary TIGER shape file from the Census Bureau [6]. We use the Geographic Coordinate System (GCS) NAD 1983 projection, which is consistent with the projection used by the Census Bureau. After this step, we are left with a total of 5,062,816 cell towers.

NHD-based filtering. The second filter uses the National Hydrography Dataset (NHD) from US Geological Survey [9]. This dataset includes features such as rivers, lakes, ponds, etc. Once again we employ the overlap tool to eliminate cell towers located in waterbodies. We are left with a total of 4,908,175 cell towers after this step. Overall, $\sim 3 \%$ of the US cell towers were eliminated as a result of the filtering steps.

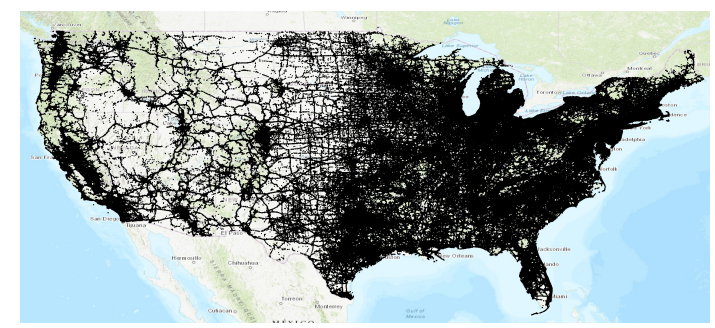

Figure 1: Locations of cell towers in the US from OpenCelliD [11].

Cell tower deployments. A geographical visualization of the cell towers in the US is shown in Figure 1. The figure highlights the density of cell towers around major population centers as well as a close correspondence to major highways across the country. It also shows higher density of cell towers in the east vs. sparser deployments in the west.

\subsection{Census Dataset}

Our analysis uses multiple US demographic datasets from the Census Bureau. These datasets consist of both shape files and tables for population estimates. We use the following shape files: (1) US outline [3], (2) Metropolitan Statistical Area (MSA) ${ }^{2}$ boundaries extracted from the Core Based Statistical Area [6], (3) Block-level population estimates from Population \& Housing Unit Counts [4], (4) Counties (and equivalent) [6], (5) Census Tracts [6], and (6) Roads [6]

Majority of the shape files listed above are not directly associated with population estimates (Block-level shape files being the only exception). In order to associate the population estimates with the areas indicated, we rely on the following Census FactFinder tables [1]: (1) Annual Estimates of the Resident Population [2], and (2) URBAN AND RURAL Universe [12].

\subsection{Data Center Locations}

Internet Atlas [19] is a repository of Internet infrastructure data gathered from public sources via web search. Data is cleaned, normalized and made openly available to the community. We obtain data center locations from Internet Atlas as the base case of our deployment model (see §3.2). The dataset consists of geographic information of 867 data centers in the US.

\section{DEPLOYMENT ANALYSIS}

\subsection{Cell Tower Proximity to Population}

3.1.1 Metropolitan Statistical Areas. We start by counting the number of cell towers deployed in MSAs. There are a total of 389 MSAs in the US. Table 1 shows the top 10 MSAs ranked based on count of cell towers deployed. To compute these counts, we use the spatial query and overlap tools in ArcGIS and proceed as follows. Given a shape file of point features (i.e., geographic locations of cell towers) and another shape file of polygon features (1.e., MSA regions shape file from the TIGER dataset) as inputs, we compute the spatial overlap between each MSA and the cell towers using the aforementioned tools to produce the count of cell towers within each MSA. Table 1 also depicts the latest (2016) annual estimate of residential population [2], area (in square mile areas) of each MSA, and the number of cell towers per person. From the table, we observe a close correspondence between top MSAs with cell tower deployments and top MSAs in terms of population densities. Due to space constraints, our analysis is focused primarily on the top 3 MSAs.

Next, we estimate the residential population covered by each cell tower in the top 3 MSAs. Spatial coverage of individual cell towers has been analyzed using Voronoi tessellations (also known as Thiessen polygons) in prior studies [16, 25]. This method does not consider antenna characteristics, but is considered a reasonable approximation for coverage of deployments. Thus, we use Voronoi cells for our cell tower coverage analysis. To facilitate this analysis, we use the Create Thiessen Polygons tool in ArcGIS, which takes geographic locations of cell towers and MSA regions as input and partitions an area into Voronoi cells. Specifically, for each cell tower location, the tool produces a Thiessen polygon such that any location within the polygon is closer (by the measure of Euclidean distance) to its associated cell tower than to any other cell tower. The tool then partitions the spatial plane into Voronoi cells and hence requires a boundary to delimit the spatial plane of interest (MSA regions shape file).

${ }^{2}$ MSAs are the most populated geographical regions in the US according to the Census Bureau [8] 
Table 1: Cell tower deployment summary statistics for top 10 MSAs.

\begin{tabular}{|c|r|r|r|r|}
\hline Metropolitan Statistical Area (MSA) & $\begin{array}{r}\text { Cell Towers } \\
\text { Count }\end{array}$ & $\begin{array}{r}\text { Population } \\
\text { (2016 estimate) }\end{array}$ & $\begin{array}{r}\text { Area } \\
\text { (square miles) }\end{array}$ & $\begin{array}{r}\text { Cell towers } \\
\text { per person }\end{array}$ \\
\hline \hline New York-Newark-Jersey City, NY-NJ-PA & 310,916 & $20,153,634$ & $9,211.62$ & 0.0154 \\
Los Angeles-Long Beach-Anaheim, CA & 248,227 & $13,310,447$ & $5,699.01$ & 0.0186 \\
Chicago-Naperville-Elgin, IL-IN-WI & 174,348 & $9,512,999$ & $9,578.60$ & 0.0183 \\
Dallas-Fort Worth-Arlington, TX & 141,100 & $7,233,323$ & $9,285.22$ & 0.0195 \\
Atlanta-Sandy Springs-Roswell, GA & 124,932 & $5,789,700$ & $8,481.03$ & 0.0216 \\
Washington-Arlington-Alexandria, DC-VA-MD-WV & 120,085 & $6,131,977$ & $6,030.38$ & 0.0196 \\
Houston-The Woodlands-Sugar Land, TX & 109,256 & $6,772,470$ & $10,071.73$ & 0.0161 \\
Miami-Fort Lauderdale-West Palm Beach, FL & 103,345 & $6,066,387$ & $6,137.05$ & 0.0170 \\
Philadelphia-Camden-Wilmington, PA-NJ-DE-MD & 100,499 & $6,070,500$ & $4,870.09$ & 0.0166 \\
San Francisco-Oakland-Hayward, CA & 91,728 & $4,679,166$ & $3,426.14$ & 0.0196 \\
\hline
\end{tabular}

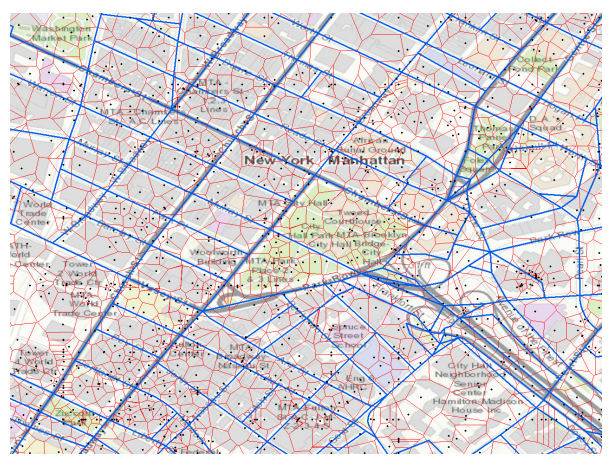

Figure 2: Cell towers (black dots), Voronoi cells (red polygons) and Census Blocks (blue polygons) in the Manhattan area of NYC.

To compute the residential population estimate served by each Voronoi cell, we use the Census Blocks dataset. A census block is the smallest geographic area for which the Census Bureau releases population estimates [5]. Figure 2 depicts the location of cell towers (black dots), the Voronoi cells computed (red polygons), and the census blocks (blue polygons) in the Manhattan area of New York City (NYC). We note that census blocks typically are larger than the Voronoi cells in MSAs (also highlighted in Figure 2). To account for this, given that we have the population estimates for each census block, we employ the following steps to determine the population estimate of each Voronoi cell:

- Compute area of each Voronoi cell (in $\mathrm{m}^{2}$ ). This step requires re-projection of the Voronoi layer using Projected Coordinate System (PCS). For consistency with the GCS used, we use NAD 1983 (2011) Contiguous USA Albers projection.

- Given a set of $N$ Voronoi cells contained within a population block $B$, compute the sum of square area of all the Voronoi cells contained within a census block as: totalArea $a_{B}=\sum_{i=1}^{N}$ area $_{i}$.

- Given the estimate for population block $B$, compute population estimate (popEst) for each contained Voronoi cell $i$ as:

$$
\text { popEst }_{i}=\text { popEst }_{B} *\left(\text { area }_{i} / \text { totalArea }_{B}\right)
$$

- If a Voronoi cell $i$ spans $M$ population blocks, compute population estimate as:

$$
\text { popEst }_{i}=\max _{1<=j<=M} \text { popEst }_{B_{j}} *\left(\text { area }_{i} / \text { totalArea }_{B_{j}}\right)
$$

We codify the above steps using the spatial join tool in ArcGIS. To classify cell towers based on their estimated residential population, we also employ the Classify numerical quantities using Graduated Colors tool in ArcGIS. Figure 3-(left) shows the residential population estimate produced by our analysis for the New York (NY) MSA. The average and median square area of Voronoi cells in NY MSA are $38,213.86 \mathrm{~m}^{2}$ and $9,707 \mathrm{~m}^{2}$. The average estimated residential population of Voronoi cells is 25 . These distributions are highly right skewed because of the presence of larger Voronoi cells closer to the boundary of the MSA. In the center of MSA, on the contrary Figure 3-(left) shows highly populated, smaller sized Voronoi cells. The right skewed nature of these distributions is observed across all of our remaining analysis. The classification tool produces 5 classes, each of which is color coded (for a range of population estimates). The classes for NY MSA range from: dark green $(0-20)$, light green (21 - 55), yellow (56 - 113), orange (114 - 238) and red (239 - 2140).

Similarly, Figure 3-(center) shows the estimated residential population per cell tower for the Los Angeles (LA) MSA. The classes for the LA MSA are: dark green (0 - 15), light green (16 - 42), yellow (43 - 87), orange (88 - 184) and red (185 - 1588). The average and median square area of Voronoi cells in LA MSA are 46,009.28 $\mathrm{m}^{2}$ and $13,341.3 \mathrm{~m}^{2}$. The average estimated residential population of Voronoi cells is 19. Figure 3-(right) shows the same analysis for the Chicago MSA. The classes for the Chicago MSA are: dark green (0 - 15), light green (16 - 45), yellow (46 - 108), orange (109 - 362) and red (363 - 759). The average and median square area of Voronoi cells in the Chicago MSA is 74,159.80 $\mathrm{m}^{2}$ and 22,477.07 $\mathrm{m}^{2}$. The average estimated residential population of Voronoi cells is 15. Overall, these results show that the correspondence between cell towers and population density is consistent in large MSAs. This analysis also provides an ability to categorize cell towers based on the residential population they serve, which forms the basis of our MEC deployment model (see §3.2).

3.1.2 Rural Area Analysis. Next, we consider cell tower deployments in less populated, rural areas. The 2010 US census [1] shows that $19 \%$ of the US population lived in rural areas ${ }^{3}$. Census data includes urban and rural population estimates for all counties (census tracts). A Census tract's population estimate is obtained by aggregating a set of Census Block Groups, which are in turn obtained by aggregating a set of Census Blocks. To identify a target rural area for analysis, we picked the census tract which had the highest rural

\footnotetext{
${ }^{3}$ The US Census Bureau has a broad definition for rural areas: areas outside urban areas and urbanized clusters
} 

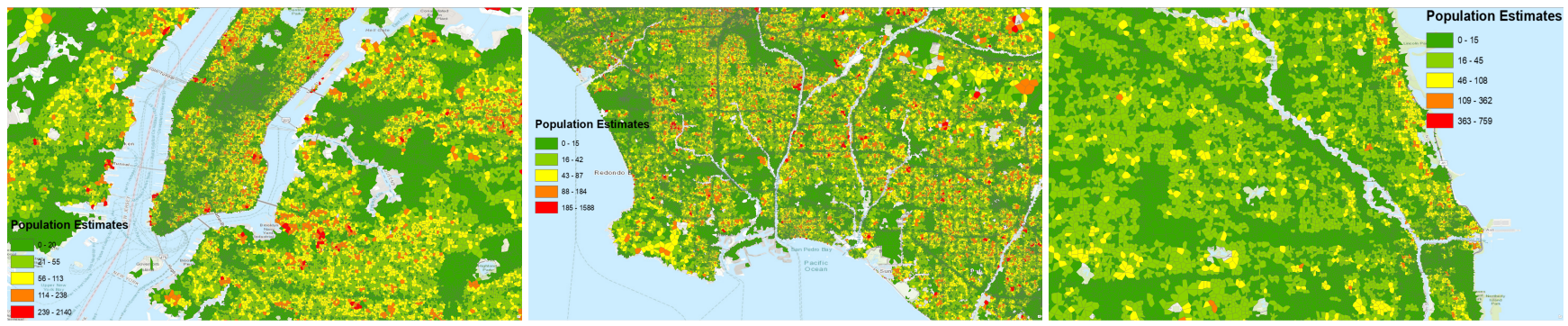

Figure 3: Per cell tower population estimate variation for NY MSA (left), LA MSA (center) and Chicago MSA (right).

population estimate count. This census tract was part of St. Clair county in Alabama.

Next, we create the Voronoi cells for towers in St. Clair county and compute population estimates using the same steps described above, with the exception of type of population dataset employed for this analysis. The population estimate from census block dataset was too fine-grained for the St. Clair county. The size of Voronoi cells and the population blocks were quite similar, but there was no direct spatial overlap between them, thereby making any meaningful estimation difficult. To consistently apply the same estimation approach as that of MSAs, we consider the census tract dataset [1]. In the census tract dataset, the population polygons are, again, larger than size of the Voronoi cells.

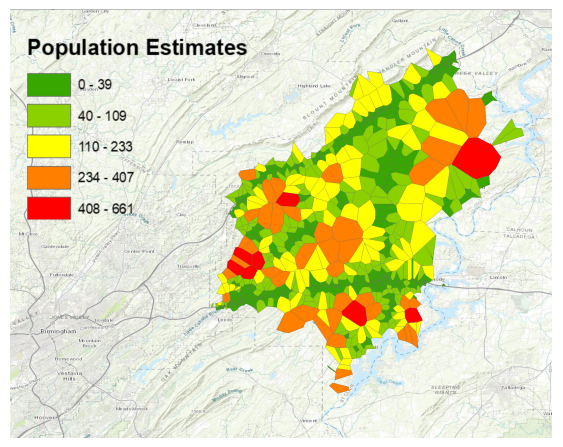

Figure 4: Per cell tower population estimate variation for St. Clair, AL Rural Area.

Figure 4 shows the result of this analysis for St. Clair county. The average square area of Voronoi cells in St. Clair county is $1,144,799.37 \mathrm{~m}^{2}$. This is much larger than the MSAs. Correspondingly, the average estimated residential population of Voronoi cells is 52 , which indicates that each cell tower serves much larger residential population than in MSAs. The classes for St. Clair county are dark green (0 - 39), light green (40 - 109), yellow (110 - 233), orange (234 - 407) and red (408 - 661), with the preponderance of dark green cells clustered along two highways that span the county.

3.1.3 Highways Analysis. Drivers on highways often rely on cell towers for GPS and other application usages. Apart from static residential population estimates, we extend our analysis by considering dynamic usage across cell towers using the Average Annual Daily Traffic (AADT) [14] counters, to account for the usage by drivers. AADT counters provide a total count of vehicles per day (averaged annually in both directions) that use a given section of a highway. We use the road shape file dataset provided by Census Bureau. In this analysis, we consider only those cell towers that are within 0.25 miles from either side of highways using Locate Features Along Route tool in ArcGIS. We consider a busy section of highway, representing traffic between two population centers for our analysis, the details of which are highlighted in the example below. Similar to MSA analysis, computation of Voronoi cells for these towers also require boundaries to delimit the spatial plane. To account for this, we use Create Polygon Features from ArcGIS to create bounding boxes around the selected sections of highways.

With the goal of estimating people driving on the section on a per-hour basis, we take an average of all the AADT values available for the chosen section of the highway. We assume that drivers are evenly distributed on the road at any time and that there is 1 driver/cell user per vehicle. Assuming that drivers are active over a 20 hour period, we divide the averaged AADT value by 20 to obtain the per-hour estimates of users served by each cell tower. Specifically, estimate for each cell tower $i$ is:

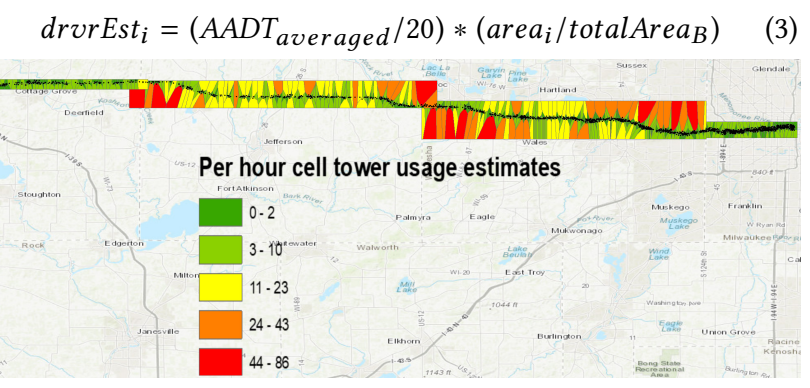

Figure 5: Per cell tower driver estimate variation for I-94 in Wisconsin.

Figure 5 shows the result of this analysis on the 70 mile section of I-94 between Madison, WI to Milwaukee, WI. The results show higher cell tower density closer to the population centers, hence fewer drivers per Voronoi cell. It also reflects that areas in between have more demand and likely do not serve a population other than drivers.

\subsection{MEC deployment analysis}

Next, we consider the problem of identifying geographic locations for deploying MEC micro data centers. We propose a methodology that considers incremental deployment of micro data centers 
to different categories of cell towers that were identified in our population density analysis above.

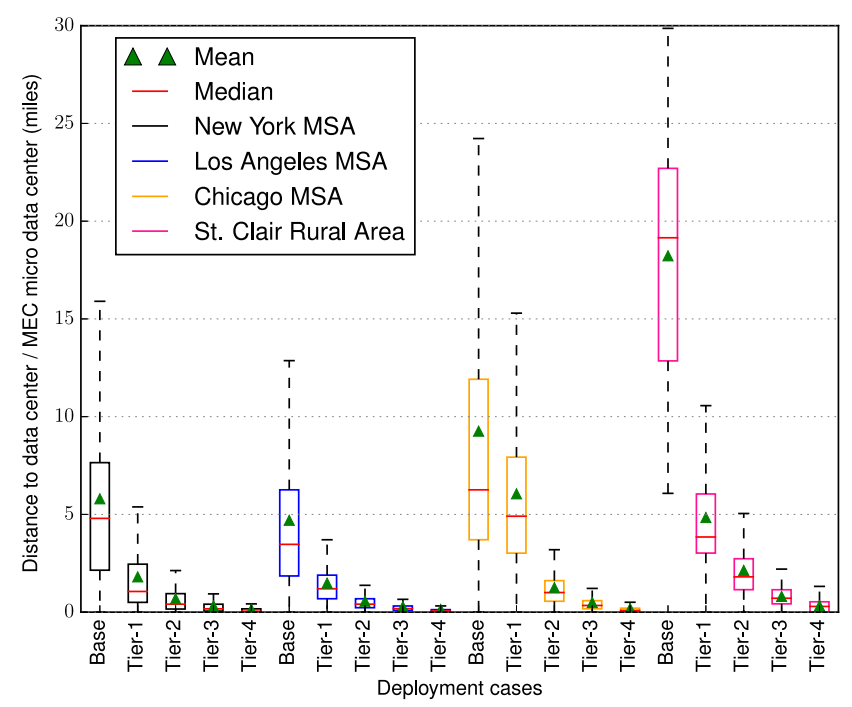

Figure 6: Box-and-whiskers plot of distances in miles to the closest data center / MEC micro data center for each cell tower.

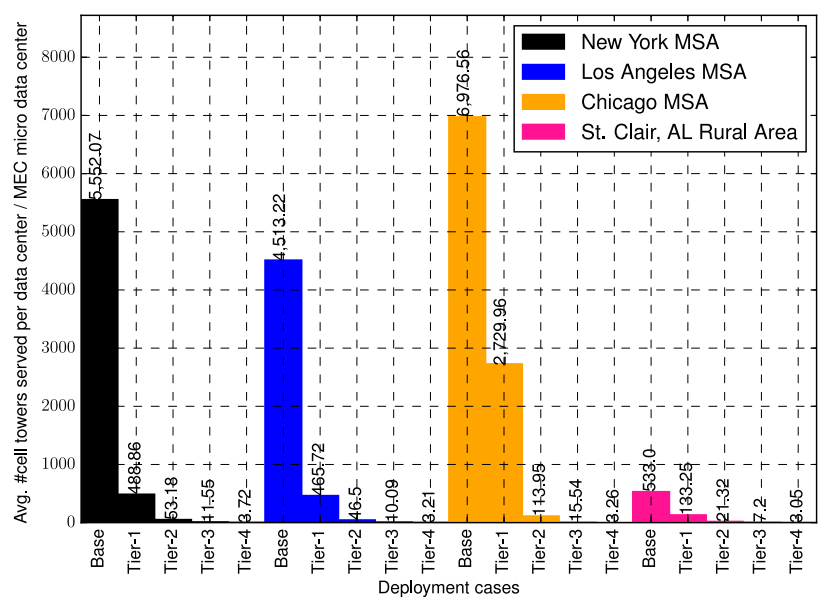

Figure 7: Average number of cell towers served per data center / MEC micro data center.

Incremental deployment model. For each of the top-3 MSA's and the rural area (St. Clair county) considered in our analysis above, we model latency as the distance of a cell tower to the closest data center. Specifically, we consider two point shape file layers: cell tower locations (merged with each tower's population estimate) and data center locations. To calculate geographical distance to nearest data center, we use the Near tool in ArcGIS, which takes the above two point shape file layers as input. We then propose an

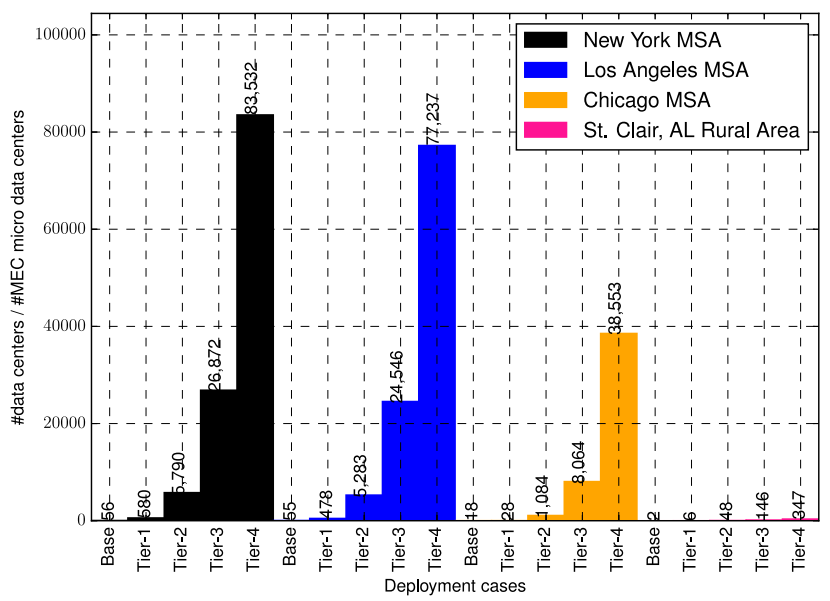

Figure 8: Number of data centers (base case) and number of micro data centers (other cases).

incremental deployment model for MEC micro data centers based on tiers of population estimate classes identified above to show the reduction in latencies achieved for each cell tower. Specifically, our analysis considers the following: (1) Base case: existing data centers from Internet Atlas dataset. (2) Tier-1 case: deployment of MEC micro data centers co-located with cell towers, which were classified in the red class. These are the towers which correspond to highest population estimates. (3) Tier-2 case: deployment of MEC micro data centers co-located with orange class cell towers. (4) Tier-3 case: deployment of MEC micro data centers co-located with yellow class cell towers. (5) Tier-4 case: deployment of MEC micro data centers co-located with light green class cell towers. Our analysis assumes that a given tower/population will be served by the data center or MEC micro data center that is geographically nearest (i.e., micro data centers act as aggregation sites).

For each deployment case, Figure 6 shows the box-and-whiskers plot of distance estimates in miles for the top 3 MSAs and the rural area. The figure highlights the reduction in distance to the nearest data center / MEC micro data center. Figure 7 shows the histogram of the average number of cell towers served per data center or MEC micro data center for each MSA and the rural area. The results show clearly the reduction of cell towers served per data center, as we consider the progressive tiers of the incremental deployment. For example, in New York MSA, adding 580 MEC micro data centers in Tier-1 deployment case, decreases the mean distance from 5.8 miles (Base case) to 1.8 miles (Tier-1 case). It also reduces the number of cell towers served per data center from 5,552.07 (Base case) to 488.86 (Tier-1 case).

Figure 8 shows the histogram of the number of data centers for the base case of each MSA and rural area. In case of the MSAs, these data centers are located within the region of the MSA. On the other hand, in the case of the rural area (St. Clair county), the 2 data centers are outside the boundary of the county (but closest to the cell towers within the county). The figure also shows the number of MEC micro data centers for the Tier- 1 to Tier- 4 cases.

To analyze the reduction in distance (to the closest data center or MEC micro data center) achieved by our deployment model, we 

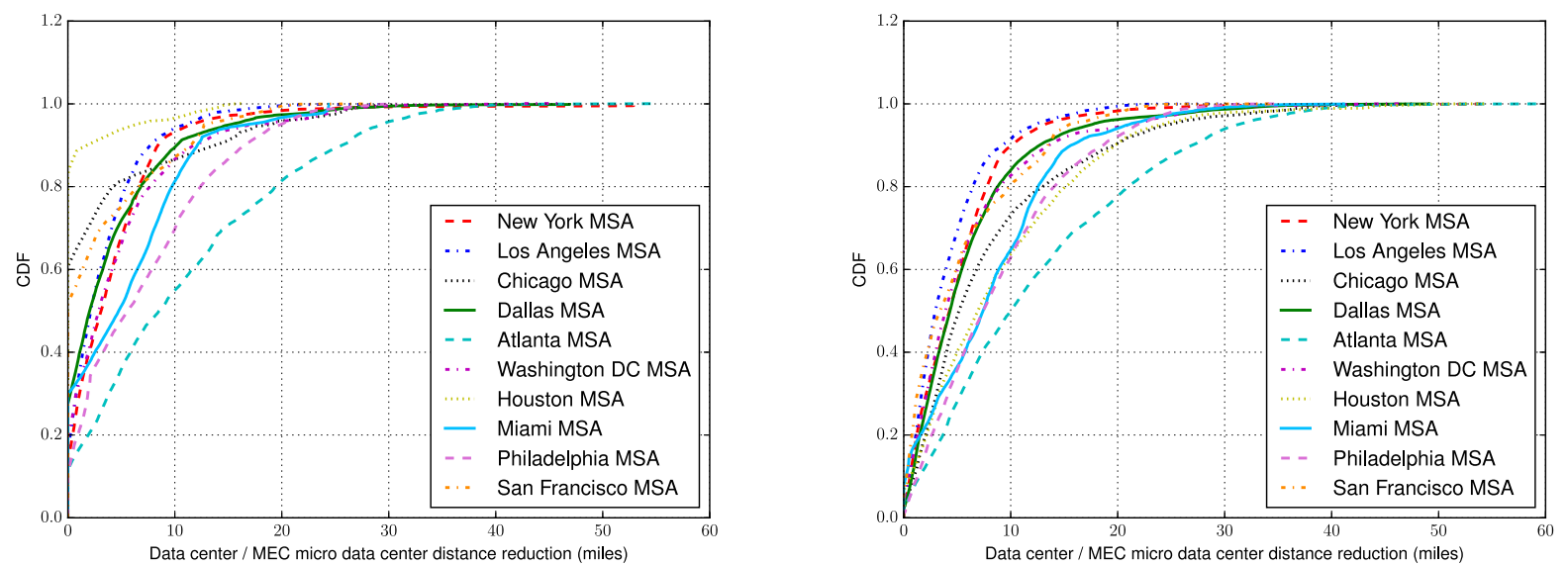

Figure 9: Per cell tower distance reduction to data center / MEC micro data center for Tier-1 case (left) and Tier-2 case (right) for top 10 MSAs.

consider the top 10 MSAs. For each cell tower within the MSA, we compute the distance to the closest data center from the Internet Atlas dataset [19]. We consider this computed distance as the base distance. Then for all the deployment tiers, we compute the distance to the closest data center, which could either be a deployed MEC micro data center or an existing data center. Finally, for each cell tower, we calculate the difference between the base distance and the distance in current tier.

Figure 9-(left) shows the CDF of distance reduction (in miles) for the Tier-1 deployment case. In the Chicago MSA, a deployment of just 28 MEC micro data centers achieves a distance reduction of greater than nearly 14 miles for at least $10 \%$ of the cell towers $(\sim 17,000$ cell towers). On average, we achieve a reduction of nearly 12 miles for approximately $10 \%$ of the cell towers across all 10 MSAs. Out of the top 10 MSAs, the Atlanta MSA shows the best distance reduction of nearly greater than 26 miles for $10 \%$ of the cell towers.

Figure 9-(right) shows the CDF of distance reduction (in miles) for the Tier-2 deployment case. Based on the design of our deployment model, the distance to the closest data center or MEC micro data center reduces from Tier-1 to Tier-2. Hence we observe an improvement in distance reduction between Tier-1 and Tier-2. Tier-2 deployment has a greater impact in MSAs like Houston, Chicago, and San Francisco. However, the distance reduction gained by Tier3 and Tier- 4 deployments are minimal, and due to space constraints we have not shown these results.

\section{RELATED WORK}

MEC offers latency and performance benefits by pushing cloud computing closer to edge of the network-a key factor driving telecoms (e.g., AT\&T plans to fuel applications such as VR, self-driving cars, etc. using MEC [10]; Verizon's MEC vision can be found in [13]). While a majority of efforts have focused on resource management and allocation [18, 20,22], load balancing [28], network architectures [27], programmability/virtualization [17], models for handling data [21], among others, one important issue stands out: site selection for MEC deployments [20,26]. To address this issue, Bouet et al. proposes a graph-based partitioning algorithm to deploy MEC servers [15], which is motivated by the spatial locality of MEC servers [24]. Zhao et al. optimizes the placement of virtual process control functions using an iterative algorithm with resilience constraints. Our work is complementary: we propose a tier-based approach by empirically studying the mobile edge infrastructure to understand their current capacity and to identify future deployments.

\section{SUMMARY AND FUTURE WORK}

Our study considers cell tower and data center locations and population distribution in the US. Using Voronoi cell-based analysis, we find that cell tower deployments are relatively consistent in large urban areas and along highways near those areas, but that deployments are more sparse and inconsistent in rural areas. We also find that cell towers are typically within 10 miles of data centers in large urban areas, but distances can be much further in rural areas. Finally, we show how micro data center deployments at cell towers can reduce distances. While our analysis is limited, we believe that it provides useful insights on MEC edge deployments and a roadmap for future GIS-based assessments. We continue to investigate edge deployment issues including considering different models for micro data center deployments, transmission range issues and how user mobility can impact MEC.

\section{ACKNOWLEDGMENTS}

This work is supported by NSF grants CNS-1703592, DHS BAA 1101, AFRL FA8750-12-2-0328. The views and conclusions contained herein are those of the authors and should not be interpreted as necessarily representing the official policies or endorsements, either expressed or implied, of NSF, DHS, AFRL or the U.S. Government. 


\section{REFERENCES}

[1] 2018. American FactFinder. https://factfinder.census.gov/faces/nav/jsf/pages/ index.xhtml.

[2] 2018. Annual Estimates of Resident Population. https://factfinder.census.gov/ bkmk/table/1.0/en/PEP/2016/GCTPEPANNR.US24PR.

[3] 2018. Cartographic Boundary Shapefiles. https://www.census.gov/geo/ maps-data/data/tiger-cart-boundary.html.

[4] 2018. Census Block Demographic Data. https://www.census.gov/geo/maps-data/ data/tiger-data.html.

[5] 2018. Census Blocks and Block Groups. https://www2.census.gov/geo/pdfs/ reference/GARM/Ch11GARM.pdf.

[6] 2018. Census Geography TIGER shape files. https://www.census.gov/geo/ maps-data/data/tiger-line.html.

[7] 2018. ESRI ArcGIS. http://www.esri.com/software/arcgis/arcgisonline/features.

[8] 2018. Metropolitan Areas. https://www2.census.gov/geo/pdfs/reference/GARM/ Ch13GARM.pdf.

[9] 2018. National Hydrography Dataset. https://nhd.usgs.gov/NHD_High_ Resolution.html.

[10] 2018. The Cloud Comes to You: AT\&T to Power Self-Driving Cars, AR/VR and Other Future 5G Applications Through Edge Computing. http://about.att.com/ story/reinventing_the_cloud_through_edge_computing.html

[11] 2018. The world's largest Open Database of Cell Towers. https://opencellid.org/.

[12] 2018. URBAN AND RURAL Universe. https://factfinder.census.gov/faces/ tableservices/jsf/pages/productview.xhtml? src=bkmk.

[13] 2018. Verizon Lays Out a Vision for Mobile, Fiber \& Cloud. https:/www.lightreading.com/mobile/5g/ verizon-lays-out-a-vision-for-mobile-fiber-and-cloud/d/d-id/733406.

[14] 2018. WisDOT interactive traffic count map. http://wisconsindot.gov/Pages/ projects/data-plan/traf-counts/default.aspx.

[15] Mathieu Bouet and Vania Conan. 2017. Geo-partitioning of MEC Resources. In Workshop on Mobile Edge Communications.

[16] Julián Candia, Marta C González, Pu Wang, Timothy Schoenharl, Greg Madey, and Albert-László Barabási. 2008. Uncovering individual and collective human dynamics from mobile phone records. Journal of physics A: mathematical and theoretical 41, 22 (2008).
[17] Kleber V Cardoso, Mohammad J Abdel-Rahman, Allen B MacKenzie, and Luiz A DaSilva. 2017. Virtualization and Programmability in Mobile Wireless Networks: Architecture and Resource Management. In Workshop on Mobile Edge Communications. ACM.

[18] Francesco De Pellegrini, Antonio Massaro, Leonardo Goratti, and Rachid ElAzouzi. 2017. Competitive caching of contents in 5G edge cloud networks. In Modeling and Optimization in Mobile, Ad Hoc, and Wireless Networks (WiOpt). IEEE.

[19] Ramakrishnan Durairajan, Subhadip Ghosh, Xin Tang, Paul Barford, and Brian Eriksson. 2013. Internet atlas: a geographic database of the internet. In 5th ACM workshop on HotPlanet. ACM.

[20] Yuyi Mao, Changsheng You, Jun Zhang, Kaibin Huang, and Khaled B Letaief. 2017. A survey on mobile edge computing: The communication perspective. IEEE Communications Surveys \& Tutorials 19, 4 (2017).

[21] Nitinder Mohan, Pengyuan Zhou, Keerthana Govindaraj, and Jussi Kangasharju. 2017. Managing Data in Computational Edge Clouds. In Workshop on Mobile Edge Communications. ACM.

[22] Abhinandan S. Prasad, Mayutan Arumaithurai, David Koll, and Xiaoming Fu. 2017. RAERA: A Robust Auctioning Approach for Edge Resource Allocation. In Workshop on Mobile Edge Communications.

[23] Joel Sommers and Paul Barford. 2012. Cell vs. WiFi: on the performance of metro area mobile connections. In IMC. ACM.

[24] Nicolas Tastevin and Mathieu Bouet. 2016. Characterizing and modeling the distance of mobile calls: A metropolitan case study. In IEEE 27th Annual International Symposium on Personal, Indoor, and Mobile Radio Communications. IEEE.

[25] David B Taylor, Harpreet S Dhillon, Thomas D Novlan, and Jeffrey G Andrews. 2012. Pairwise interaction processes for modeling cellular network topology. In IEEE GLOBECOM.

[26] Tuyen X Tran, Mohammad-Parsa Hosseini, and Dario Pompili. 2017. Mobile edge computing: Recent efforts and five key research directions. IEEE COMSOC MMTC Commun.-Frontiers.

[27] Shuo Wang, Xing Zhang, Yan Zhang, Lin Wang, Juwo Yang, and Wenbo Wang. 2017. A survey on mobile edge networks: Convergence of computing, caching and communications. IEEE Access 5 (2017)

[28] Ye Yu, Xin Li, and Chen Qian. 2017. SDLB: A Scalable and Dynamic Software Load Balancer for Fog and Mobile Edge Computing. In Workshop on Mobile Edge Communications. ACM. 